\title{
Secondary Task Method for Workload Measurement in Alarm Monitoring and Identification Tasks
}

\author{
Xiaojun $\mathrm{Wu}$ and Zhizhong $\mathrm{Li}^{*}$ \\ Department of Industrial Engineering, Tsinghua University, Beijing, 100084, China \\ zzli@tsinghua.edu.cn
}

\begin{abstract}
The operators who perform alarm monitoring and identification tasks in complex industrial systems, such as nuclear power plants, often suffer from heavy mental workload. Among the many existing mental workload measurements, secondary task method was chosen and examined for that it is sensitive to operator capacity, provides a common metric for different tasks, and easy to carry out, etc. In this study, a paper-based arithmetic secondary task was adopted to measure mental workload of the participants when they were performing alarm monitoring and identification tasks. A two-factor, two-level lab experiment was conducted to examine whether this secondary task method can discriminate different levels of mental workload, and whether it interferes with the primary task. The results showed that though the secondary task chosen in this experiment had good discriminability, its influence on the primary task performance and its interaction with scenario complexity were significant.
\end{abstract}

Keywords: Mental workload, secondary task, alarm monitoring, alarm identification.

\section{Introduction}

Modern industrial systems, such as nuclear power plant (NPP) and air flight control systems, are becoming more and more complex, and can often bring heavy mental workload to their operators (Hwang, et al, 2008). Therefore, it is very important to find out how high is the level of operators' mental workload when they are performing tasks such as alarm monitoring and identification.

Mental workload is characterized as a mental construct that reflects the mental strain which is resulted from performing a task under specific environmental and operational conditions, coupled with the capability of the operator to respond to those demands (Cain, 2007). Though there is no universally acceptable definition of mental workload, it has been generally defined as the amount of resource difference between task demands and capacity provision by an individual (Sanders and McCormick, 1987).

Hwang et al. (2008) divided the existing methods to measure mental workload into three categories: subjective ratings, performance measures, and physiological measures.

\footnotetext{
* Corresponding author.
} 
For measuring the mental workload in alarm monitoring and identification tasks in the main control room (MCR) of a NPP, the pros and cons of these three different categories are summarized as follows.

It is widely claimed that the subjective measures have advantages such as inexpensive, unobtrusive, easily administered, widely transferable, and having high face validity (Casali and Wierwille, 1983; Wickens, 1984). However, it has shortcomings such as potential confounding of mental and physical workload, dissociation of subjective ratings and task performance, and raters' different interpretation of scale words, etc. (O’Donnell and Eggemeier, 1986; Meshkati et al., 1990). For alarm monitoring tasks which might experience fluctuations during a time period, the dependence of subjective measures on short-term memory might distort the workload rating for that period (O'Donnell and Eggemeier, 1986).

There are two categories of performance measures, including primary task performance measures and secondary performance measures. Primary task performance measures provide direct indications for interested task performance. However, they are not very sensitive to changes of workload, especially when operators have spare capability to increase their effort level. In this sense, primary task performance measures are not appropriate to be used in NPP MCRs. Secondary task method is one of the mostly widely used methods to measure workload, for that it provides a sensitive measure of operator capacity, a sensitive index of task impairment due to stress, and a common metric for comparison of different tasks (Gawron, 2008). However, it has one major disadvantage: the possible intrusion on the performance of the primary task (Willigs and Wierwille, 1979). As for the alarm monitoring and identification tasks, if we can find a secondary task that has little intrusion on the performance of the primary task, then it might provide us with continuous, objective, and direct measure of mental workload.

Physiological measures use the known features sensitive to operator loadings indicators to measure mental workload (Wierwille, 1979). The main advantage of physiological measures is that they can provide continuous and objective data of the operator's state. Usually, physiological measures mainly focus on the cardiovascular system, respiratory system, nervous system and biochemistry, with heart rate variability being probability the mostly widely used one (Hancock, 1988). As physiological measures have to explain the effect of workload through the psychological process happening on the body, they are indirect. Thus, it would provide a more complete understanding of the workload when combined with other workload assessment techniques. Besides, some physiological measures are invasive and will affect the performance of primary tasks (such as the respiratory system), while some of them require participants to stay still for reliable data collection (such as the nervous system), which is not appropriate to be used in NPP MCRs.

Currently, secondary task methods have been used to measure mental workload in aviation and vehicle driving domains (Wierwille et al, 1977; Williges and Wierwille, 1979; Casali, and Wierwille, 1983; Blanco et al., 2006). However, there are few studies which use secondary task methods to measure operators' mental workload in control rooms where monitoring and identification tasks are performed. Previous studies have showed that properly designed secondary tasks can be used to estimate the workload of primary task (Wierwille, 1977), while there existed some evidences that secondary tasks differ in the degree to which they interfere with the primary task, 
and they may also interact with the primary tasks, with the greatest interference and interaction occurring when the primary and secondary tasks involve similar processes or rely on similar brain regions (Troyer, 1999).

This study attempts to use the paper-based arithmetic secondary-task method for workload assessment of alarm monitoring and identification tasks in NPP MCRs. When operators are performing alarm monitoring and identification tasks, they should periodically look up the operating procedures to check whether the current parameter value exceeds its normal range or they should periodically put down some critical process parameters on paper for future filing. The arithmetic secondary task introduced in this study is different from the tasks that operators perform in the real situation. We want to examine whether this kind of secondary task can discriminate the different levels of operators' mental workload under different cases, and whether the secondary task we introduced will interfere with the performance of the primary task.

There were two hypotheses in this study. (1) The paper-based arithmetic secondary task method can discriminate the simple scenarios from the complex scenarios, with more correctly answered arithmetic calculations in simple scenarios than in complex scenarios. (2) The arithmetic secondary task does not interfere with the primary tasks, nor does it interact with the primary tasks. A two-factor, two-level within-subjects experiment design was adopted to test the above two hypotheses.

The methodology used in this study is described in Section 2. Section 3 reports the experiment results. Section 4 presents some discussion on the results. Conclusions from this study are given in Section 5.

\section{Methodology}

\subsection{Participants}

In total, 15 paid male students of engineering background from Tsinghua University participated in the experiment. Only male students were recruited to keep the gender consistency as that in NPP MCRs. The participants aged from 20 to 24 (Mean=21.73, $\mathrm{SD}=1.39$ ), all had more than 5 years' experience with computers and were thus familiar with computer operations, but had very limited knowledge about nuclear power plants.

\subsection{Independent Variables and Experiment Design}

A two-factor within-subjects design was adopted in this study. The first factor was the secondary task. Each participant performed half of the task scenarios with the secondary task, and the other half of the task scenarios without the secondary task. The sequence of the two halves was randomized to minimize the learning effect. The second factor was complexity of task scenarios, which had two levels: simple scenarios with six alarms in two minutes, and complex scenarios with twelve alarms in two minutes. There were six simple task scenarios and six complex task scenarios. The order of the simple and complex scenarios were randomized and in an alternate manner. 


\subsection{Experiment Task}

When an alarm was triggered, a participant pressed the button named 'Alarm Discovered' to report that he noticed the alarm and to press the button of the alarm that has been triggered to suppress the alarm. Then the participant made judgment about to which system component that the alarm was associated with and how severe it was. Both the correctness of alarm detection, and errors made when making alarm judgments were recorded for further analysis.

Each participant carried out the alarm monitoring and identification tasks for 12 scenarios in total. A paper-based arithmetic secondary task was introduced in half of the scenarios (six in total) during the experiment to measure the mental workload of the participants. The secondary task required the participants to perform two digits addition calculation. The secondary tasks were performed on paper, while both the alarm monitoring and identification task was performed on the computer. The participants were asked to do the secondary tasks as many as they could while trying their best not to affect the performance of the primary tasks.

\subsection{Dependent Variables}

Objective performance measurements were collected for hypothesis testing, including correctness of alarm detection (the ratio between the number of alarms correctly perceived and the total number of alarms presented), time to detect an alarm (the time interval between the moment when the alarm was triggered and the moment when participant pressed the button 'Alarm Discovered'), error rate of alarm identification (the ratio between the number of errors made in alarm identification and the total number of alarms triggered), time to identify an alarm (the time interval between the time when a participant suppressed the alarm and the time when the participant made an identification of the alarm), and performance of the secondary task which indicates mental workload (the number of correctly answered arithmetic operations).

\subsection{Experiment Platform and Experiment Procedure}

The experiment was conducted on a personal computer with two screens. The program was coded with Visual Basic 2010, and the scenario data was contracted from the PCtran ${ }^{\mathrm{TM}}$ simulation software, which can simulate different working conditions of a pressurized water reactor (PWR) NPP.

Fig. 1 illustrates one of the experimental displays. The alarms were divided into three severity levels. The Level 1 alarms indicate the plant wide, most severe ones, and were indicated with the red color to attract the attention of the participants. Level 2 alarms were system wide functional failures, which should be timely dealt with, and were indicated with the hotpink color. Level 3 alarms were parameter level malfunctions, the lower than normal range alarms were indicated with blue bars, which grew to the left side of the neutral position as a parameter value was decreasing, while the 
higher than normal range alarms were indicated with yellow bars, which grew to the right of the neutral position as a parameter value was increasing. All alarms kept flashing until they were suppressed by a participant.

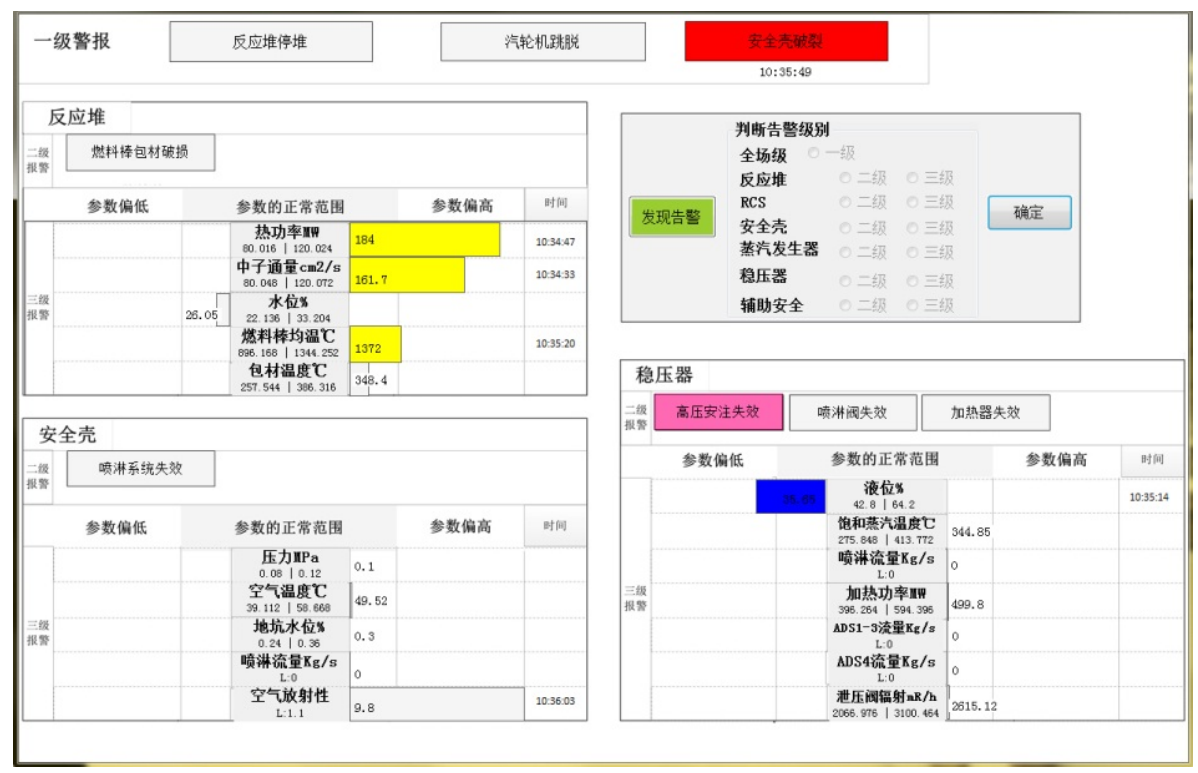

Fig. 1. Illustration of an alarm display

The experiment task lasted about 90 minutes for each participant. Before a participant began the experiment, his demographic information was collected and an informed consent was signed voluntarily. At the beginning, the participant was given a 30-minute training session about the NPP working principals and the usage of the alarm system. Then, the participant took a session of 20 -minute practice to get familiar with the alarm system. Finally, the formal test in 12 scenarios was performed, lasting about 40 minutes.

\section{$3 \quad$ Results}

\subsection{Mental Workload Measurement}

The results listed in Table 1 show that the introduced arithmetic secondary task can discriminate the simple cases from the complex ones $($ sig. $=0.000<0.05)$ in the number of correctly answered arithmetic calculations, but not in the error rate of arithmetic calculations (sig. $=0.256>0.05$ ). This means that when using the secondary task method, the number of correctly answered arithmetic calculations could be considered as a suitable secondary task performance criterion to reflect the workload of participants. 
Table 1. Comparison of performance of the secondary task between simple and complex cases

\begin{tabular}{|c|c|c|c|c|c|}
\hline \multirow{2}{*}{$\begin{array}{l}\text { Performance of } \\
\text { secondary task }\end{array}$} & \multicolumn{2}{|c|}{ Simple cases } & \multicolumn{2}{|c|}{ Complex cases } & \multirow[t]{2}{*}{ Sig. } \\
\hline & Mean & $\mathrm{SD}$ & Mean & $\mathrm{SD}$ & \\
\hline $\begin{array}{l}\text { No. of correctly answered } \\
\text { arithmetic calculations }\end{array}$ & 19.022 & 10.644 & 6.667 & 5.382 & $0.000^{*}$ \\
\hline $\begin{array}{l}\text { Error rate of arithmetic } \\
\text { calculations }\end{array}$ & 0.0109 & 0.0112 & 0.0193 & 0.0285 & 0.256 \\
\hline
\end{tabular}

*: The difference is significant, with sig. $<0.05$.

\subsection{Interference with Primary Task Performance}

The effect of the secondary task on the performance of the primary tasks was analyzed. The results are summarized in Table 2. It turns out that the introduction of the secondary task significantly reduced the correctness of alarm detection (sig. $=0.000<$ 0.05 ), prolonged the time to detect an alarm (sig. $=0.017<0.05$ ), and shortened the time to identify an alarm $(\mathrm{sig} .=0.000<0.05)$. As for the error rate of alarm identification, no significant effect was found.

Table 2. Influence of secondary task on primary task performance

\begin{tabular}{|c|c|c|c|c|c|c|c|c|}
\hline \multirow[t]{2}{*}{ Task } & \multirow[t]{2}{*}{$\begin{array}{l}\text { Performance } \\
\text { criteria }\end{array}$} & \multicolumn{2}{|c|}{$\begin{array}{l}\text { Without } \\
\text { secondary } \\
\text { task }\end{array}$} & \multicolumn{2}{|c|}{$\begin{array}{l}\text { With second- } \\
\text { ary task }\end{array}$} & \multirow{2}{*}{$\begin{array}{l}\text { Sig. of } \\
\text { sec- } \\
\text { ondary } \\
\text { task }\end{array}$} & \multirow[t]{2}{*}{$\begin{array}{l}\text { Sig. of } \\
\text { com- } \\
\text { plexity }\end{array}$} & \multirow[t]{2}{*}{$\begin{array}{l}\text { Sig. of } \\
\text { Inte- } \\
\text { raction }\end{array}$} \\
\hline & & $\begin{array}{c}\text { Mea } \\
\mathrm{n}\end{array}$ & $\mathrm{SD}$ & Mean & SD & & & \\
\hline Alarm & Correctness of & 0.99 & 0.01 & 0.95 & 0.000 & $0.000^{*}$ & 0.095 & $0.001^{*}$ \\
\hline Moni- & alarm detection & 5 & 8 & 8 & & & & \\
\hline toring & $\begin{array}{l}\text { Time to detect an } \\
\text { alarm }\end{array}$ & 3010 & 1299 & 3559 & 1225 & $0.017^{*}$ & $0.000^{*}$ & $0.005^{*}$ \\
\hline Alarm & Error rate of & 0.02 & 0.02 & 0.02 & 0.034 & 0.948 & 0.952 & 0.163 \\
\hline $\begin{array}{l}\text { iden- } \\
\text { tificat }\end{array}$ & $\begin{array}{l}\text { alarm identifi- } \\
\text { cation }\end{array}$ & 3 & 7 & 2 & & & & \\
\hline ion & $\begin{array}{l}\text { Time to identify } \\
\text { alarm }\end{array}$ & 2989 & $\begin{array}{c}483 . \\
2\end{array}$ & 2711 & 506.6 & $0.000^{*}$ & $0.019^{*}$ & 0.788 \\
\hline
\end{tabular}

*: The difference is significant, with sig. $<0.05$.

The complexity of scenarios had significant effects on both the time to detect an alarm $($ sig. $=0.000<0.05)$ and the time to identify an alarm $($ sig. $=0.019<0.05)$, but had no significant influence on correctness of both alarm monitoring and identification.

There existed a significant secondary task * complexity interaction in alarm monitoring performance criteria, as Fig. 2 shows, but no significant interaction in alarm identification performance criteria. The introduction of the secondary task led to decreased correctness of alarm detection in both simple and complex cases, but the impairment in simple cases was much more severe than that in the complex cases 
(sig. $=0.001<0.05)$. As for the time to detect an alarm, it increased slightly in complex cases, while increased dramatically in simple cases (sig. $=0.005<0.05$ ), because of the introduction of the secondary task. This means that the negative effects by the secondary task on performance of the primary tasks in simple cases was more significant than that in complex cases.

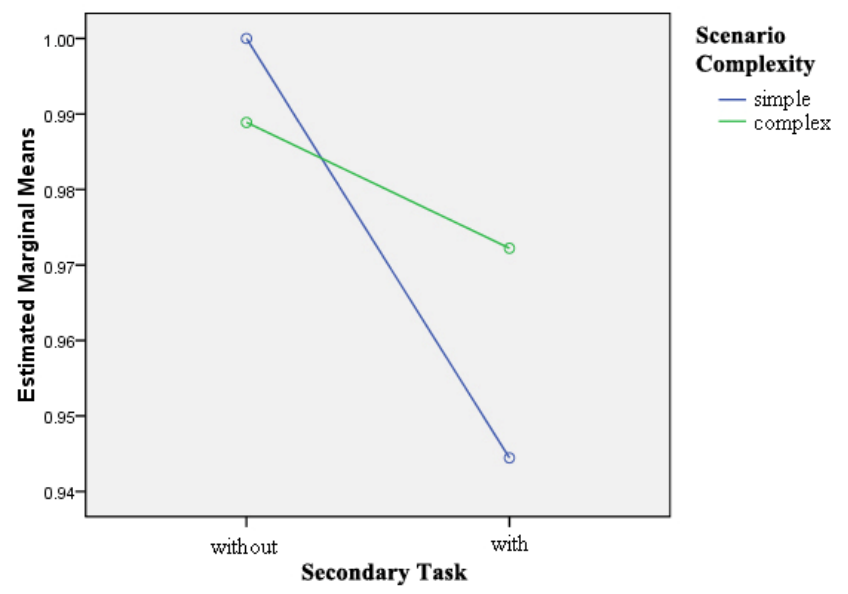

(1) Correctness of alarm detection

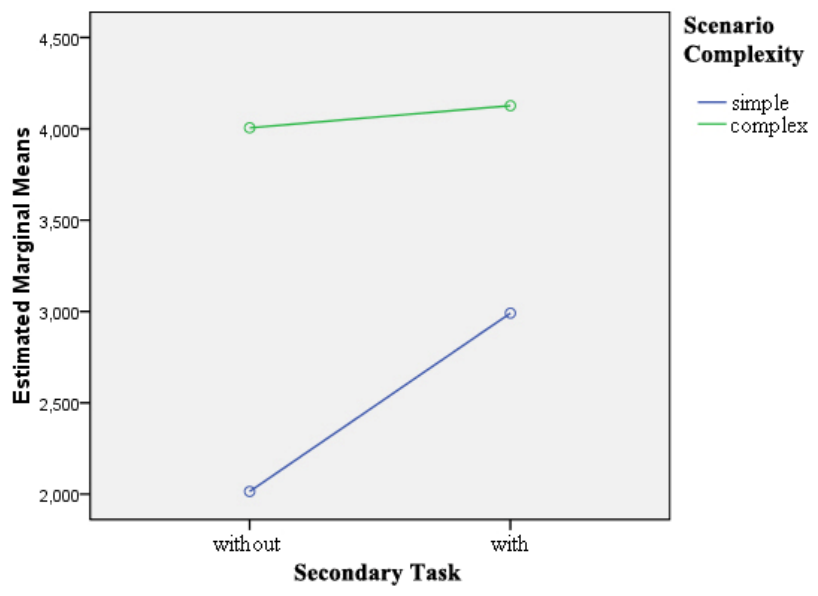

(2) Time to detect alarm

Fig. 2. Interaction effects of secondary task and scenario complexity 


\section{Discussion}

From the results, it may be concluded that the introduced paper-based arithmetic secondary task can quantitatively discriminate different levels of mental workload.

However, by comparing the primary task performance with and without the secondary task, it is found that the introduction of the secondary task significantly affected the primary task performance. The alarm detection behavior was distorted in lower correctness and longer time of alarm detection, while the alarm identification behavior was distorted in shorter time to identify an alarm. As the participants had to allocate their attention and effort between the introduced secondary task and the primary task, the promptness and correctness of alarm detection might thus be negatively affected. Once an alarm was noticed, because of the existence of secondary task, the participants rushed their alarm identification process, causing the shorter time used to identify the system component that the alarm belonged to and how severe it was.

The interaction between secondary task and scenario complexity indicates that the introduction of the secondary task had more severe negative effect on simple cases than on complex cases. Very possibly the participants adopted a different strategy in the allocation of their attention and effort between the primary and secondary tasks under the two scenario complexity levels. When the scenario was complex, the participants focused on the primary tasks, while when the scenario is simple, they switched their emphasis to the performance of the secondary task.

Though the paper-based arithmetic secondary task seemed be able to discriminate different levels of mental workload, because of its negative effect on primary task performance and its interaction with scenario complexity, it may not be suitable for the measurement of mental workload in alarm detection and identification tasks. The ideal secondary task should not introduce negative effects on the primary task performance, nor should it interact with both the primary task and other factors in a study (Troyer, 1999).

\section{Conclusions}

In this experimental study, the effect of the paper-based arithmetic secondary task on alarm monitoring and identification task was examined. It was found that the arithmetic secondary task could provide objective, continuous and direct data about mental workload, but it interfered with the primary task, and interacted with scenario complexity.

The findings from this study are interesting. Although the arithmetic secondary task has been widely adopted for mental workload measurement in domains such as driving and aviation, it might not be appropriate for the domain of alarm monitoring and identification. This would encourage us to look for more appropriate methods for the measurement of mental workload in alarm processing tasks.

Acknowledgement. This study was supported by the National Natural Science Foundation of China (Project No. 70931003). 


\section{References}

1. Blanco, M., Biever, W.J., Gallagher, J.P., Dingus, T.A.: The impact of secondary task cognitive processing demand on driving performance. Accident Analysis \& Prevention 38(5), 895-906 (2006)

2. Cain, B.: A review of the mental workload literature. Defence Research and Development Toronto, Canada (2007)

3. Casali, J.G., Wierwille, W.W.: A comparison of rating scale, secondary task, physiological and primary-task workload estimation techniques in a simulated flight task emphasizing communications load. Human Factors 25, 623-642 (1983)

4. Gawron, V.J.: Human performance, workload and situation awareness measures handbook, 2nd edn. CRC Press, Boca Raton (2008)

5. Hancock, P.A., Meshkati, N. (eds.): Human mental workload, vol. 52. North-Holland (1988)

6. Hwang, S.L., Yau, Y.J., Lin, Y.T., Chen, J.H., Huang, T.H., Yenn, T.C., Hsu, C.C.: Predicting work performance in nuclear power plants. Safety Science 46(7), 1115-1124 (2008)

7. Meshkati, N., Hancock, P.A., Rahimi, M., Dawes, S.M.: Techniques in mental workload assessment. In: Wilson, J.R., Corlett, E.N. (eds.) Evaluation of Human Work: A Practical Ergonomics Methodology, 2nd edn. Taylor \& Francis, Philadelphia (1995)

8. O'donnell, R.D., Eggemeier, F.T.: Workload assessment methodology. Measurement Technique 42, 5 (1986)

9. Sanders, M.S., McCormick, E.J.: Human factors in engineering and design. McGRAWHILL book company (1987)

10. Troyer, A.K., Winocur, G., Craik, F.I., Moscovitch, M.: Source memory and divided attention: Reciprocal costs to primary and secondary tasks. Neuropsychology 13(4), 467 (1999)

11. Wickens, C.D.: Engineering psychology and human performance. Charles E. Merril., Columbus (1984)

12. Wierwille, W.W., Gutmann, J.C., Hicks, T.G., Muto, W.H.: Secondary task measurement of workload as a function of simulated vehicle dynamics and driving conditions. Human Factors: The Journal of the Human Factors and Ergonomics Society 19(6), 557-565 (1977)

13. Wierwille, W.W.: Physiological measures of aircrew mental workload. Human Factors: The Journal of the Human Factors and Ergonomics Society 21(5), 575-593 (1979)

14. Williges, R.C., Wierwille, W.W.: Behavioral measures of aircrew mental workload. Human Factors 21, 549-574 (1979) 